\title{
Adaptive Mesh Method Applied to Poisson Solver Module for 3D Capacitively Coupled Plasma Discharge Simulation
}

\author{
Received May 15, 2020; accepted May 29, 2020
}

\author{
Sora Lee ${ }^{a}$, Yejin Shon ${ }^{a}$, Dong-gil Kimª, Deuk-Chul Kwon ${ }^{b}$, and Hee Hwan Choe ${ }^{a, *}$ \\ aSchool of Electronics and Information Engineering, Korea Aerospace University, Goyang 10540, Republic of Korea \\ ${ }^{b}$ Plasma Technology Research Center, National Fusion Research Institute, Gunsan 54004, Republic of Korea
}

\section{*Corresponding author E-mail: choehh@kau.ac.kr}

\begin{abstract}
In plasma discharge simulation, the configuration of a mesh has a significant influence on convergence. However, it is time consuming to obtain a suitable mesh. We thus propose a module approach that automatically adjusts a mesh at a specific location as the calculation proceeds. It was confirmed that the mesh is adjusted in a direction that reduces the probability of an error. The adaptive mesh refinement and coarsening method was applied to the Poisson equation module of a three-dimensional capacitively coupled plasma equipment model for semiconductors. By comparing the respective results of the adaptive mesh and fixed mesh models, we confirmed that the error of the electric potential value decreases.
\end{abstract}

Keywords: Adaptive mesh, Poisson's equation, Plasma, Asymmetric 3D capacitively coupled plasma

\section{Introduction}

Plasma discharge technology has been applied in various fields, such as semiconductor, display, energy, and environmental industries [1]. The importance of equipment that is suitable for plasma production has increased with the development of ultra-fine process technology in semiconductor processing [2]. Plasma equipment has been developed using various plasma sources-including capacitively coupled plasma (CCP), inductively coupled plasma (ICP), and electron cyclotron resonance (ECR) - based on a plasma generation method. In this paper, the most common CCP discharge source is discussed. Owing to its relatively simple structure, and because it can obtain density and uniformity, CCP has been extensively used and studied [3-5]. A model of the CCP reactor that is predominantly used in the semiconductor manufacturing process is depicted in Fig. 1. It consists of two parallel metal plates. An electric field is formed between the

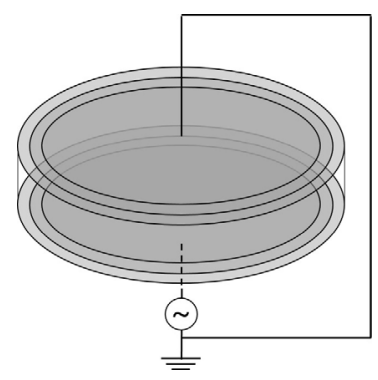

Figure 1. Schematic diagram of the capacitively coupled plasma model electrodes by radio frequency voltage that is applied from the outside. Accordingly, uniform plasma can be produced with relative ease.

To develop and apply processing equipment that is optimized for the required procedures such as dry etching and plasma-enhanced chemical vapor deposition, it is necessary to study the association of certain variables that exhibit plasma characteristics. Computational modeling has been used to understand the complex associations among these plasma parameters. If the simulation results could be efficiently analyzed by modeling, they could be applied to an efficient approach for reducing costs, processing time, and equipment development. In addition, computational modeling can identify correlations among the plasma process elements that are difficult to experimentally obtain. It thus serves as an effective means of elucidating plasma phenomena [6]. The plasma equipment that is employed in semiconductor processing frequently assumes two-dimensional (2D) axis symmetry. However, because the chamber used in the actual process is not axially symmetrical, we performed in this study a three-dimensional (3D) simulation to emulate a scenario that more closely approximates the actual process.

Our plasma simulation model uses the following plasma equation based on the fluid model, which is known as the fastest and easiest to analyze [7]. Continuous equations, electron energy equations, and drift-diffusion approximations are used for electronic modeling with Eqs. (1) to (3).

$$
\frac{\partial n_{e}}{\partial t}+\nabla \cdot \vec{\Gamma}_{e}=R_{e}
$$




$$
\begin{gathered}
\frac{\partial}{\partial t}\left(\frac{3}{2} n_{e} k_{B} T_{e}\right)+\nabla \cdot\left(\frac{5}{2} \vec{\Gamma}_{e} T_{e}-\frac{5}{2} \frac{e n_{e} k_{B} T_{e}}{m_{e} \nu_{e N}} \nabla T_{e}\right) \\
-e \nabla V \cdot \vec{\Gamma}_{e}+P_{e, \text { loss }}=P_{\text {ind }} \\
\vec{\Gamma}_{e}=\mu_{e} n_{e} \nabla V-D_{e} \nabla n_{e}
\end{gathered}
$$

where $n_{e}$ is the electron density, $\vec{\Gamma}_{e}$ denotes the electron flux, $m_{e}$ represents the electron mass, and $T_{e}$ is the electron temperature. In addition, $\nu_{e N}$ denotes the electron collision frequency with neutrals, $\mu_{e}$ is the electron mobility, $D_{e}$ represents the electron diffusivity, $k_{B}$ is the Boltzmann constant, $e$ is the electron charge, and $V$ is the potential. Moreover, $R_{e}, P_{e, \text { loss }}$, and $P_{i n d}$ respectively denote the difference between the generation and loss of electrons by reactions, the variable related to the energy in the collision process, and the absorption power due to the inductive discharge. Therefore, $P_{i n d}$ is not considered in the CCP model.

The equations of the continuity and drift-diffusion approximations for ionic species can be described as

$$
\begin{gathered}
\frac{\partial n_{i}}{\partial t}+\nabla \cdot \vec{\Gamma}_{i}=R_{i} \\
\vec{\Gamma}_{i}=\mu_{i} n_{i} \nabla V-D_{i} \nabla n_{i}
\end{gathered}
$$

where $n_{i}$ is the ion density, $\vec{\Gamma}_{i}$ denotes the ion flux, $\mu_{i}$ represents the ion mobility, $D_{i}$ is the ion diffusivity, and $R_{i}$ denotes the difference between the generation and the loss of ions by the reactions.

The electrostatic potential is calculated by solving Poisson's equation by the following method:

$$
\nabla^{2} V=-\frac{e}{\varepsilon_{0}}\left(\sum_{i} Z_{i} n_{i}-n_{e}\right)
$$

where $\varepsilon_{0}$ is the vacuum permittivity.

Figure 2 shows the relationship between Eqs. (4), (5), and (6). The potential obtained using Poisson's equation affects the flux and density of the electrons and ions. The potential obtained by Poisson's equation is used in the electron, neutron, and ionic equations, and the solutions of the equations are then used in Poisson's equation. Therefore, it is important to accurately obtain the solution for the latter equation, which has the greatest influence on the plasma equation analysis.

The mesh plays an essential role in calculating the electrical potential in terms of space and time. If the mesh size is small, the

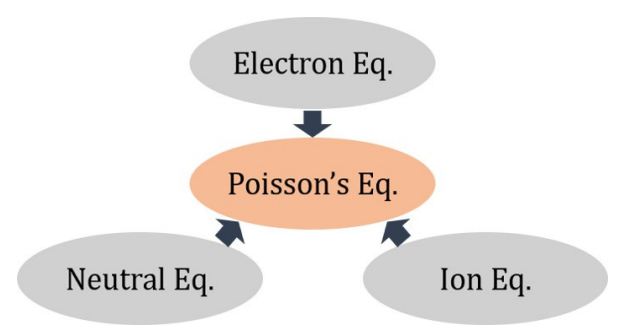

Figure 2. Diagram for depicting the relevance of the plasma equation. number of electrons per mesh unit decreases, whereas the electron temperature increases. However, if the mesh size is large, the probability of a numerical error increases; therefore, a limit exists to the mesh size control. Moreover, the time step must be less than the dielectric relaxation time. Therefore, it is necessary to carefully consider the mesh according to the time step to obtain an accurate solution.

In this study, we focused on the mesh size during the calculation of Poisson's equation. Additionally, we applied the finite element method to improve the calculation efficiency [8], which implied that several constraints exist for the geometric shapes. Therefore, even complex models could be calculated. The performed 3D simulation required significant computational resources and was highly dependent on the mesh and thus sensitive to it. An adaptive mesh was therefore applied to resolve the problems of this simulation. A fixed mesh is typically used in plasma simulations [9] because it can be easily applied for calculations. Although this approach is advantageous, the probability of an error increases when the variable range in the mesh is large. Furthermore, when the range is small, a loss of computational resources occurs. To address this issue, commercial tools for plasma simulation have applied adaptive mesh refinement to increase the accuracy. However, as the refinement method is applied to the mesh, the mesh size decreases as the calculation is repeated, and the analysis errors likewise increase.

Based on the deal.II $\mathrm{C}^{++}$library, we therefore calculated Poisson's equation by applying a method of automatically adjusting the mesh size to the CCP structure according to the variable range in the element. To reduce the dependence of the solution on the mesh, a method of refining and coarsening the adaptive mesh was studied.

\section{Model description}

This study focused on the core module used in semiconductor device processing to simulate the CCP discharge. To verify the necessity of the core module, a 3D structure model was applied. The conceptual structure of the equipment is displayed in Fig. 3. The chamber height and lower electrode diameter are 50 and $190 \mathrm{~mm}$, respectively. The upper and lower electrodes are marked in light blue and have a diameter of $150 \mathrm{~mm}$. The ceramic dielectrics surrounding these electrodes are marked in yellow and have a width of $20 \mathrm{~mm}$. The gas port through which the gas flows is marked in green and has a width of $20 \mathrm{~mm}$. The boundary condition of the lower ceramic dielectric is ground, and the boundary condition of the gas port is the Neumann boundary condition. In addition, the boundary condition between the upper surface, including the electrode, ceramic dielectric, and side wall of the chamber, is ground. $100 \mathrm{~V}$ was applied to the lower electrode at a frequency of $13.56 \mathrm{MHz}$.

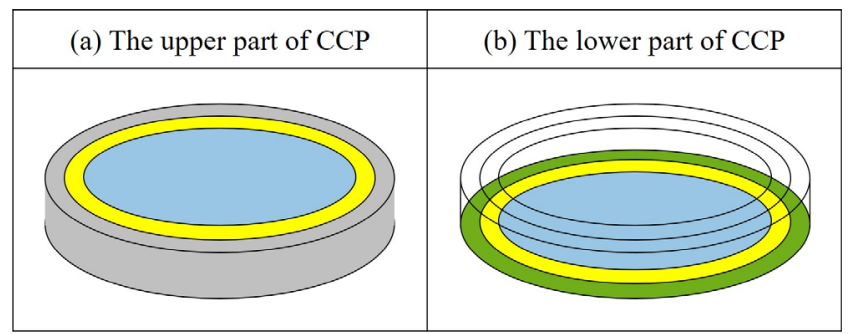

Figure 3. Schematic of the CCP simulated in this study. 
As mentioned earlier, it is essential to efficiently solve Poisson's equation for plasma discharge analysis. It is well known that the equation closely relates to the density and momentum of electrons and places numerous restrictions on the calculation [12-15]. However, when obtaining a variable that changes with time, it may be advantageous for the mesh to be variable rather than fixed to enable an effective solution. If the calculation is performed on a fixed mesh, the position varies at the point where the amount of computation required for convergence changes. In several cases, an error occurs because the computational condition for convergence is not satisfied at a specific location. It is time consuming to again find the optimized mesh by modifying the conditions for convergence or by repeating the mesh adjustment. Therefore, from a numerical and efficiency perspective, this problem can be solved if an adaptive mesh is effectively applied. In this study, we therefore focused on this aspect. We tested a module that applies a variable mesh for efficient calculation. The objective was to identify a key factor to improve the computational efficiency in conjunction with other plasma analysis equations in the future.

\subsection{Adaptive mesh modeling}

The goal of mesh adaptation is to identify the optimal mesh number and size to produce an accurate solution. This technique adjusts the accuracy of the analysis according to the analysis error. Thus, we propose a method to efficiently solve Poisson's equation by applying adaptive mesh refinement and the coarsening algorithm in the 3D CCP structure. The error estimation method is used for finer mesh segmentation, and the employed scale depends on the diagonal faces and polynomial degrees of adjacent cells [16]. The estimated error for each cell is returned and 'passed' to refine and coarsen the functions. Among the refining and coarsening functions, the most readily accessible methods are the fixed number and fixed fraction methods. In the case of a fixed number, a flag is specified to refine and coarsen a given fraction of all the cells. This refines a given fraction of all cells to provide a predictable growth in the mesh size. The fixed fraction method calculates the threshold value, compares it with the input fraction of the error total, and sets the flag to refine and coarsen the cell. This controls the reduction in error estimates. The refining and coarsening of the mesh proceeds is based on the designated flag level. When refining and coarsening is $2 \mathrm{D}$ or $3 \mathrm{D}$, a part of mesh is divided into four or eight sections, respectively. In applying the above approach, we obtained the refining and coarsening results by adjusting the fraction values of these functions to determine the optimized mesh.

\section{Results and discussion}

To demonstrate the reliability and efficiency of a simulation using an adaptive mesh, Poisson's equation was simulated in the CCP structure. A sinusoidal wave was applied to the lower electrode, and the Neumann boundary condition was applied to the ceramic region. The calculation was performed with a fixed initial mesh. Figure 4 displays the result of the phase change when one period of the applied sinusoid is T. In this figure, the maximum and minimum values of the sinusoidal wave are applied in the case of $1 / 4 \mathrm{~T}$ and $3 / 4 \mathrm{~T}$ phases. Consequently, the difference in values occurs at the boundary between the ceramic and the electrode, where the boundary condition is $\mathrm{V}=0$. When the number of meshes is constant, it is confirmed that the surface is broken near the boundary where the difference in the values

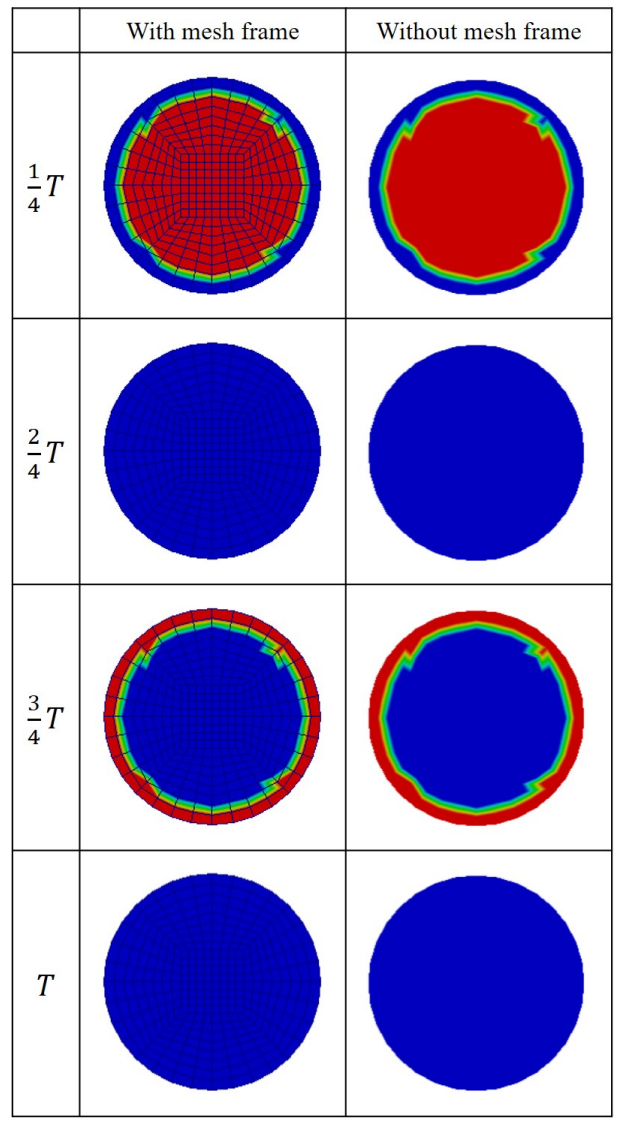

Figure 4. Results using the fixed mesh.

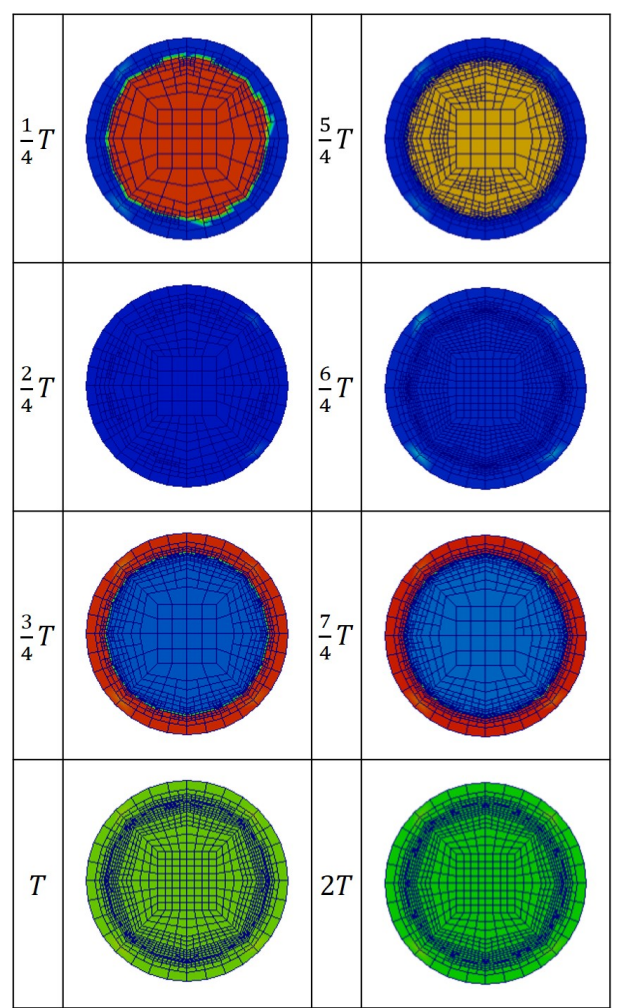

Figure 5. Results using the fixed fraction method. 
is increased.

Figure 5 presents the results of applying the fixed fraction method. As the adaptive refining and coarsening method is applied, it is confirmed that the difference between the values is refined near the boundary, whereas the other part is coarsened. In this case, the initial mesh begins with the same mesh configuration as the fixed mesh mentioned earlier. It is confirmed that the mesh configuration changes as the calculation is performed. In addition, the cells with large errors are refined in the early stages, and accurate values are obtained from the refined cells as the cycle progresses. Figure 6 displays the results of applying the fixed number method. In this case, the calculation is performed with the mesh of the fixed mesh model in the initial state. The adaptive mesh stabilizes earlier in the period than the fixed fraction method, and it is calculated in a fixed state when the appropriate number of meshes is reached.

We studied the results of calculating Poisson's equation from a controlled mesh by applying a fixed-state mesh, fixed-fraction method, and fixed-number method. Figure 7 compares these three cases at $1 / 4$

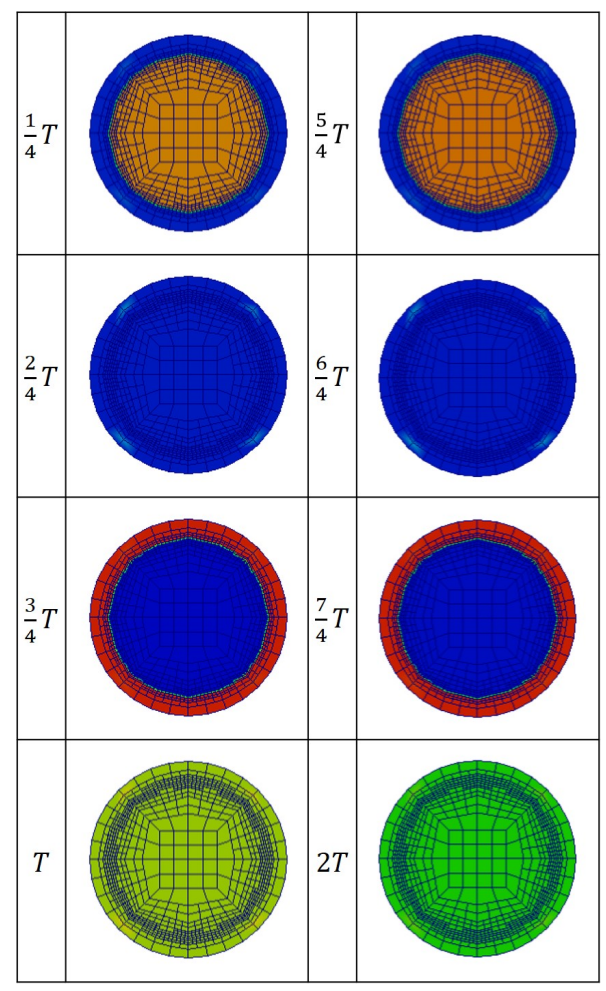

Figure 6. Results using the fixed number method.

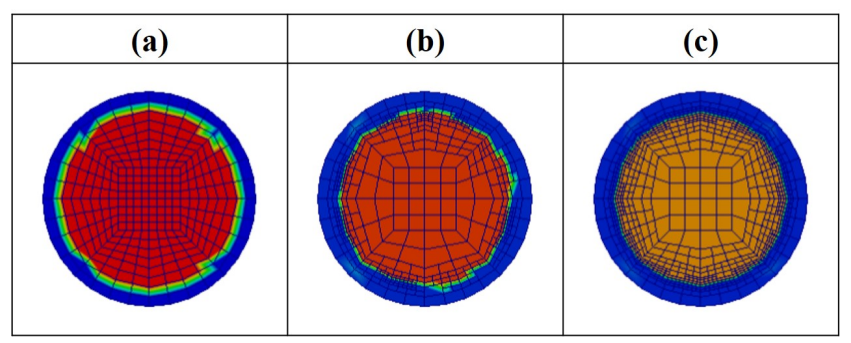

Figure 7. Results of 1/4 cycle. (a) Fixed mesh. (b) Fixed fraction method. (c) Fixed number method.
$\mathrm{T}$, where the maximum value of the sine wave is applied. First, (a) the fixed mesh exhibits a distinct difference from (b) and (c) at the boundary between the lower electrode and the ceramic. As the error increases near the boundary where the value change is large, the mesh is finely adjusted. However, coarsening was applied because the error was reduced in a relatively small or constant value change. When comparing (b) and (c), (c) is flagged to refine and coarsen all the cell fractions; hence, the number of meshes increases before (b). Therefore, it is confirmed that the stabilization of refining and coarsening proceeded rapidly and was more efficient.

\section{Conclusions}

In this study, we identified a method for calculation optimization using a variable mesh by applying adaptive mesh refinement and a coarsening algorithm using the deal.II $\mathrm{C}^{++}$library. Based on the structure of the CCP equipment used in the semiconductor manufacturing process, the proposed method was applied to the Poisson equation calculation module in the plasma discharge simulation. When a fixed mesh was applied, the probability of an error increased when the variable change was large; when the change was small, computational resources were wasted. Therefore, it was necessary to develop an adaptive mesh to improve the computational efficiency in the simulation. The adaptive mesh refining and coarsening method was used to adjust the mesh at an appropriate ratio using the respective fixed fraction and fixed number methods. The effect on the calculation of Poisson's equation was analyzed. It was confirmed that, compared to the model that assumed a fixed mesh, in the model with the adaptive mesh the result of electric potential was calculated in the direction of reducing errors in the cells with a large amount of electric potential change. It is expected that the method applied to the module in this study will improve the calculation efficiency of the whole plasma equation, and it will be the continued focus of a future study.

\section{Acknowledgements}

This work was supported by the National Research Council of Science \& Technology (NST) grant by the Korean government (MSIP) (No. CAP-20-02-NFRI).

\section{References}

[1] M. A. Lieberman and A. J. Lichtenberg, Principles of Plasma Discharges and Materials Processing (Wiley, New York, 1994).

[2] T. Makabe and Z. Petrović, Plasma Electronics: Applications in Microelectronic Device Fabrication (Taylor and Francis, New York, 2006).

[3] Y. Ohtsu, Physics of High-Density Radio Frequency Capacitively Coupled Plasma with Various Electrodes and Its Applications, In Plasma Science and Technology - Basic Fundamentals and Modern Applications (IntechOpen, London, 2018).

[4] L. L. Alves and L. Marques, Plasma Phys. Control. Fusion 54, 124012 (2012).

[5] E. Kawamura, A. J. Lichtenberg, M. A. Lieberman, and A. M. Marakhtanov, Plasma Sources Sci. Technol. 25, 035007 (2016).

[6] R. W. Hockney and J. W. Eastwood, Computer Simulation Using Particles (CRC Press, Boca Raton, FL, 1996).

[7] J. D. Bukowski and D. B. Graves, J. Appl. Phys. 80, 2614 (1996). 
[8] A. C. Polycarpou, Introduction to the Finite Element Method in Electromagnetics (Morgan \& Claypool, San Rafael, CA, 2006).

[9] S. Rebiai, H. Bahouh, and S. Sahli, IEEE Trans. Dielectr. Electr. Insul. 20, 5, 1616-1624 (2013).

[10] D. Arndt, W. Bangerth, T. C. Clevenger, D. Davydov, M. Fehling, D. Garcia-Sanchez, G. Harper, T. Heister, L. Heltai, M. Kronbichler, R. Kynch, M. Maier, J. Pelteret, B. Turcksin, and D. Wells, J. Numer. Math. 27, 4 (2019).

[11] T. Leicht and R. Hartmann, Error Estimation and HP-Adaptive Mesh Refinement for Discontinuous Galerkin Methods (World Scientific Publishing, Singapore, 2011).
[12] P. L. G. Ventzek, T. J. Sommerer, R. J. Hoekstra, and M. J. Kushner, Appl. Phys. Lett. 63, 605 (1993).

[13] P. L. G. Ventzek, R. J. Hoekstra, and M. J. Kushner, J. Vac. Sci. Technol. B: Microelectron. Nanometer Struct. Process. Meas. Phenomen. 12, 461 (1994).

[14] E. P. Hammond, K. Mahesh, and P. Moin, J. Comput. Phys. 176, 402-429 (2002).

[15] G. J. M. Hagelaar and G. N. W. Kroesen, J. Comput. Phys. 159, 1$12(2000)$.

[16] M. Ainsworth and J. T. Oden, Comput. Methods Appl. Mech. Eng. 142, 1-88 (1997). 